\title{
Design and Production of Beads Woven Fabric in Fashion Trends for Garments of Egyptian Origin
}

\author{
Elsayed Ahmed Elnashar* \\ Kafrelsheikh University, Egypt
}

*Corresponding author: Elsayed Ahmed Elnashar, Professor Textiles \& Apparel, Kafrelsheikh University, Egypt.

Received Date: November 15, 2019

Published Date: December 03, 2019

\section{Abstract}

Objectives: The continuity and revival of the heritage and raise the level of artistic taste, and to be inherited from the artworks a source of innovation to produce works of art serving different areas, to become a community of traditional Egyptian arts beads distinctive that emphasizes self-belonging to the environment for fashion trends.

Methodology: The article followed the descriptive, analytical and experimental approach. This article was limited to the study of Egyptian clothes for traditional beads woven fabric in fashion trends for garments of Egyptian origin.

Data sources and methods of collection: The field study was conducted using a number of sources to obtain the most accurate and objective article data. These included: personal interview, observation, and systematic recording of data and information through codification, photography and illustrations obtained through the above, get it from the engravings and experience the different ways to make sure it is correct.

Results and discussion: The structure of beads is the most common. The beads are made of natural materials that were within easy reach of the hands and made from the silt available on the surface of the earth for easy formation and drying. Beads were used in all cultures and at all times during different historical eras. It had a fascinating effect across the globe. And its various purposes, it was important in decoration and decoration, as was a manifestation of wealth. The structural structure of the beadwork was one of the ancient works of ancient civilizations. Such as necklaces and wide belts that covered the chest and buttocks of ancient Egyptian women. The pharaohs used beads as individual units or as a composite set in decorative units. The beads industry has been considered valuable skill since ancient times. This craft was secreted for centuries. In the history of mankind, man invented the various ways of decoration using beads until he reached the same developments in the industry, where he made in large quantities and from many different raw materials and in innovative forms with advanced techniques for his importance in modern fashions.

Keywords: Beads woven fashion trends; Garments; Egyptian origin

\section{Introduction}

Traditional fashion in Egypt is an area in which women have developed and diversified styles of decoration and performance. And the most important resort to Egyptian women in the decoration of her clothes, embroidery, which many types and forms and raw materials and methods of performance, and found alone, as found with other materials such as beads. In this study, it sheds light on the various types of crafts and their uses, as well as the ways and means of their implementation. And to identify the uses of beads and its materials and types and techniques for the implementation of various types of crafts, which include: (beadwork on the surface of the cloth - single beadwork. Then the methods of finishing the various pieces of pottery were shown in cilia or blocks.
Beads woven made of row upon row of cylindrical beads, often with wristlets, anklets, and shoulder straps of matching design. Made and shoulder straps of matching design. However, artists who were representing their clients for eternity were not interested in the latest fashions and were usually reluctant to portray them. The rich tomb owner is never showing wearing bracelets like those of Djer, nor of Queen Hetep-heres, the wife of Sneferu and mother of the builder of the Great Pyramid; the queen`s bracelets, of heavy gold hoops with an inlaid design of brightly colored butterflies. Amulets, furthermore, are scarcely ever shown, although they were the commonest type of jewelry and existed by the thousands. Of various shapes and materials, and each with its own specific role, 
they were usually tired around the finger, wrist, or neck by a piece of string; the esteem in which they were held by their owners is reflected in a story that has come down to us from the age of the pyramids [2] (Figure 1).

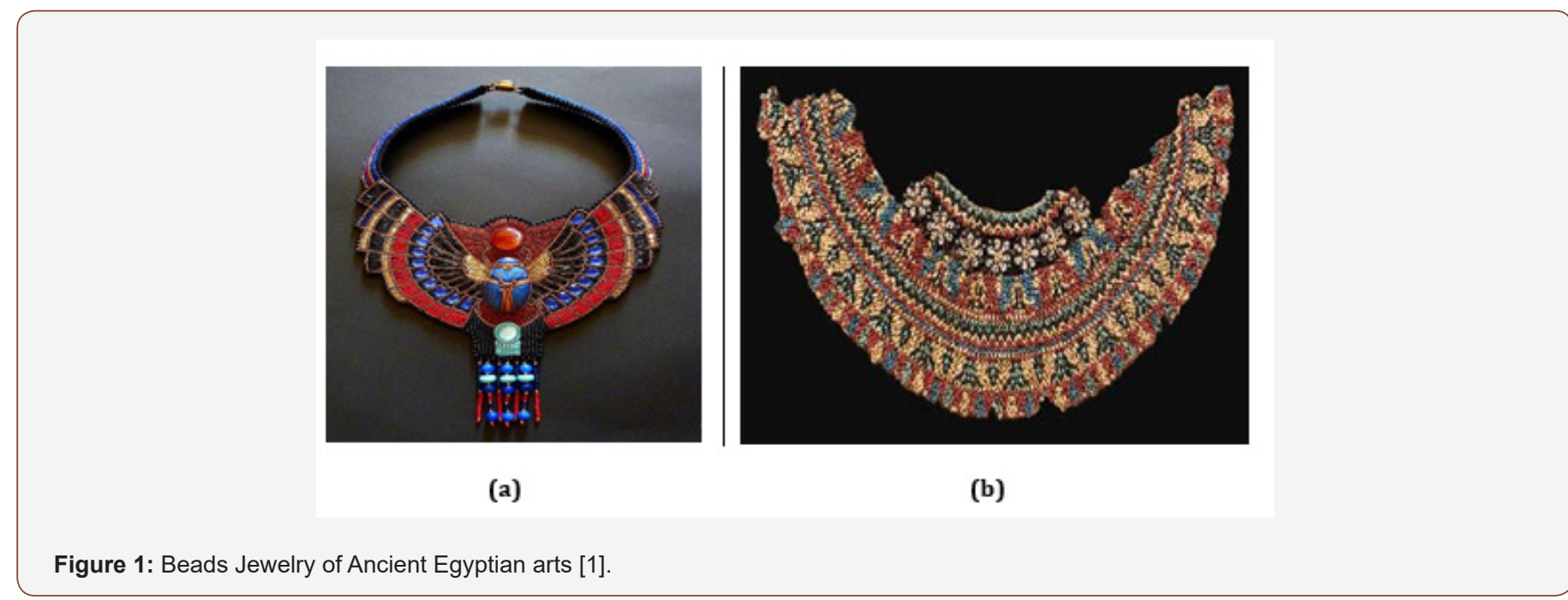

These beautiful beading patterns demonstrate how simple stitching patterns can be used to create a woven tapestry of beads that is utterly entrancing to the eye, that means beads woven fabric that all you die-hard wirework fans and chainmaille devotees be warned because these gorgeous designs might just bring beads woven fabric over to the beading side.

\section{Beads Definitions}

Is a form punctured by a hole or holes can be organized through it, and is composed of multiple ores, whether natural ore such as some fruits, plant seeds, deposit, shells, wood, ivory, glass and pottery, plastic, wood, ivory, pearl and also beads are made of metals such as gold Silver and copper or of industrial and natural materials together as gilded beads is considered the first glass base in the ore beads. The colors of the beads are numerous, as well as being bright, transparent or opaque. It also takes a variety of forms such as rectangle, cube, spherical, rhombus, oval, cylindrical and hexagonal or any irregular shape. The size ranges between large, medium, small and very small and uses beads in decorating and beautification, which is It has various specifications according to its material that makes it fit with other materials such as cloth, thread, metal, and leather. The beads are colored either by placing the colors with the material of the beads, this is known as dye in the paste, or coloring at a later stage so that the color above the original material of the beads.

\section{Beads methods techniques}

- $\quad$ Style beads systems

- Multi-style systems multi-style beads systems with wire

- Weave style beads

- $\quad$ Perhaps the easiest and simplest methods used is the first method, and fits more with computer embroidery machines, which is the systems of beads in a thread of nylon hinge thickness depending on the size of the hole of the beads, and through this technique can get innovative embroidery

\section{Materials of Beads Woven Fabric}

There are many types of beads, including glass beads, metal, wood, and beads made from marine products such as snails, shells, pearls, corals, the article community is limited to the use of types of beads: glass beads, metal beads, snails, seeds. Glass beads: There are many types of glass beads. In traditional society, women use a small type known as Rocailles beads. Rocailles is a French word for small stones. This type of beads was known thousands of years ago in the Venetian era and used traditional methods known to date. It was also known in traditional societies as pound beads and round beads because it was sold in pounds. This type of beads is the most suitable types of glass beads for embroidery, so the Bedouin woman was keen to use it and instinct to use it is characterized by its uniform and equal size with a simple shine granular, and is characterized by its low price for other types of beads this in addition to the availability in the market and the diversity and beauty of its colors. This type of beads is divided into a number of types according to its color opaque stone beads: It is the most used species in different cultures and in traditional societies in general and in the research community in particular so that it is known as traditional beads is a small glass beads opaque color enters into the glass paste itself shows solid color and prefer this type in traditional societies because It retains its color and does not lose it during use and is characterized by the plain colors preferred by traditional communities. Transparent glass beads: a transparent bead line the hole from the inside color and these colors are given by the color and rarely used in the research community, perhaps because of the demise of color with use.

Metallic glass beads: It is a transparent glass beads lining the inside of the hole with a golden or silver metal layer giving it a golden or silver color or metallic luster colors has been used in traditional clothing in recent times instead of metal beads, because of its availability and cheapness.

Pearl glass beads: It is an opaque glass beads with light colors characterized by a special shine resembles the pearl. 
Glass beads rainbow color: It is a glass beads multiple colors and gives one color of the colors of the rainbow and is not used in the research community.

Long glass beads (Najaf output): a type of glass beads, but it is long (tubular), and appeared a few in the Bedouin of the south.

Crystal lobes: Some traditional clothes have recently been added round lobes of crystal.

\section{Glass Beads Whole Techniques}

Small beads are perforated from one side, and large beads may be perforated from either side, or formed around a metal axis and then removed, leaving holes in place. In the pulled and blown glass beads, the hole is just an air bubble. With the technical development, the electric drill as well as the laser was used to puncture the beads.

\section{Metal beads}

Metals are involved in the manufacture of this type of beads, such as gold, silver, platinum, copper and zinc. They may be mixed to make certain alloys to obtain different shapes and types The Article community was limited to the use of lead beads and silver and copper beads.

\section{Lead beads}

This type has been widely used in the Egyptians region. One of the most important types known and prepared it by melting the lead and then dipping the thorn of the thorns or a needle in the molten lead amount of it on the head of the fork and then dipped in water, and is withdrawn from the fork by human. This type is characterized by its strength and hardness and also available in the local markets a small type of lead beads known, which is cheap compared to casting.

\section{Silver Beads}

It is a small beads or core of silver or has a buttonhole (perforated ring) and is known in the search area silver buttons.

\section{Copper beads}

The copper beads were not used in the sense, but empty bullets were used after firing them, the back is removed, resulting in a pierced tube from both sides used as a copper bead, used to beautify the ends of the women's leather belt in the southern region.

\section{Design and Design of Chenille}

The popular folklore has become more and more popular in all parts of the world. Folk costumes [3-5] methods of implementation, decoration and colors are an art of the arts, with its components, methods and integrated elements that make it a special unit, close to human life. The traditional Egyptian fashion was an area in which women developed and varied styles of decoration and performance. The patterns of technology varied in spite of the primitive means and tools, so that these costumes and their decorations acquired a distinctive creativity that reflected the ability to use available in the surrounding environment for purposes beyond the components of the material itself. A way to express their own distinct taste. The most important thing that Egyptian women resorted to in the decoration of their clothes is the embroidery, which has many types, shapes, materials and methods of performance, and found the embroidery alone, as found with other materials such as beads.

\section{Threads}

Liberated silk or cotton embroidery threads are used, in the form of a paralysis analysis is known. The threads with beads give a beautiful decorative shape, where women sometimes resort to fill some spaces between the beads with embroidery stitches and therefore, they are complementary to the work of beads.

\section{Decorative units}

Bead motifs were based on simple geometric decorative motifs such as straight, parallel and intersecting lines, refracted (zigzag), square, rhombus, rectangle, circle and triangle.

Sections and techniques of beadwork: -Beadwork can be divided into types in terms of apparent form: A): Beadwork on the surface of the cloth. B): Beadwork on the ends without a cloth background, used in the covers of the face and head and some other household items. C): Individual beadwork, a system in different decorative ways may be added to the clothing pieces, or some supplements such as rosary, or used as pendants. D): Woven beadwork such as belts, headband, and shoes, and fashion clothes, we can use of beads in a weaving method is one of the most durable, most wellknown, and easy-to-learn methods. Two methods of weaving beadwork were followed: the plain $1 / 1$ weaving method and the Weft Twining method: Egyptian women excelled in many parts by choosing raw materials, colors and decorations, and the multiplicity and innovation of techniques and that she is an instinctive artist where she has many artistic foundations in her works. We would like to take advantage of these products now in a manner suitable for modern fashion; Addressing heritage becomes a rich source of innovative design and artwork.

\section{Conclusion}

The implementation methods differed according to the decorative needs and the method of using the piece.

\section{Acknowledgement}

None.

\section{Conflict of Interest}

Authors declare no conflict of interest.

\section{References}

1. Beads Jewelry of Ancient Egyptian arts (2019)

2. Scott NE (1964) The Metropolitan Museum of Art Bulletin Egyptian Jewelry.

3. Elnashar EA (2018) A Unified Fashion Technology in Trends of Textile Engineering Science 7000Bc in Egypt. Trends in Textile Engineering \& Fashion Technology 2(1): 139-141.

4. ElNashar EA, Zlatev Z, Ilieva J (2016) Textile Patterns Based on Ancient Egyptian Ornaments. ARTTE 4(2): 92-104.

5. ElNashar EA, Zlatev Z (2016) Ancient Egyptian Ornaments for The Contemporary Fashion. Innovation and entrepreneurship applied scientific journal 4(3): 1-5. 